\title{
Rapid diagnosis of asymptomatic hereditary haemochromatosis by detection of the Cys282Tyr mutation in the HLA-H gene
}

\author{
S Aslam, GR Standen
}

\begin{abstract}
Summary
Hereditary haemochromatosis is an autosomal recessive disorder characterised by life-long excessive accumulation of iron. A candidate gene for hereditary haemochromatosis has recently been reported (HLAH) and a specific missense mutation (Cys282Tyr) has been identified in $85 \%$ of patients with the disorder. We describe the rapid detection of this mutation using the polymerase chain reaction and restriction endonuclease digestion. The usefulness of this test for early diagnosis of hereditary haemochromatosis in asymptomatic family members is highlighted.
\end{abstract}

Keywords: haemochromatosis, iron accumulation, polymerase chain reaction

Hereditary haemochromatosis $(\mathrm{HH})$ is a common autosomal recessive disorder characterised by a life-long excessive accumulation of iron. ${ }^{1}$ Iron deposition in cardiac, hepatic and endocrine tissue leads to tissue damage and, if untreated by venesection, the disease has high morbidity and mortality from cardiomyopathy, diabetes mellitus, cirrhosis, and hepatoma. The condition is one of the commonest genetic abnormalities with a carrier frequency estimated at about $5-10 \%$ in Caucasians and a homozygote frequency of 1 in 200-400. Previous studies have shown linkage of the putative $\mathrm{HH}$ gene with the HLA locus on chromosome $6 \mathrm{p}$ and a significant association is found with the HLA-A3 antigen. The mechanism responsible for the excessive iron accumulation is unknown.

A candidate gene (HLA-H) for $\mathrm{HH}$ has recently been identified and $85 \%$ of patients were found to be homozygous for a specific missense mutation, Cys282Tyr. ${ }^{2}$ The carrier frequency in the normal population was $6.4 \%$. The authors detected the mutation by the polymerase chain reaction (PCR) using an allele-specific oligonucleotide-ligation assay. We describe the detection of the mutation by PCR using simple endonuclease digestion and show how the test can be employed to confirm the diagnosis of $\mathrm{HH}$ in an asymptomatic patient with early evidence of iron accumulation.

\section{Case report}

A 49-year-old pre-menopausal woman (P1) was referred for investigation of possible $\mathrm{HH}$. She was asymptomatic apart from lethargy and mild arthralgia. There were no abnormal findings on clinical examination. Her 55-yearold brother had previously been diagnosed as having $\mathrm{HH}$ on the basis of liver dysfunction, grossly elevated serum ferritin $(>2000 \mu \mathrm{g} / \mathrm{l})$, raised serum iron and reduced total ironbinding capacity (transferrin saturation: $60 \%$ ). He refused to undergo a liver biopsy. Initial investigation of patient $\mathrm{P} 1$ showed normal liver function, serum ferritin $217 \mu \mathrm{g} / 1$ (normal range for pre-menopausal women : $15-120 \mu \mathrm{g} / \mathrm{l}$ ), serum iron $27.6 \mu \mathrm{mol} / 1$ (normal range: $13-32 \mu \mathrm{mol} / \mathrm{l}$ ), total iron-binding capacity $46.9 \mu \mathrm{mol} / 1$ (normal range: $45-$ $70 \mu \mathrm{mol} / \mathrm{l}$ ) and transferrin saturation $59 \%$.

\section{DNA analysis}

Genomic DNA was isolated from patient P1's leukocytes by standard methods and amplification of the segment of the HLA-H gene containing the Cys282Tyr mutation was performed using the oligoprimers described by Feder et $a l^{2}$ :

Sense:

5' TGGCAAGGGTAAACAGATCC 3'

Antisense:

\section{5' CTCAGGCACTCCTCTCAACC 3'}

The PCR conditions were initial denaturation at $94^{\circ} \mathrm{C}$ for $5 \mathrm{~min}$, and then 30 cycles of $94^{\circ} \mathrm{C}$ for $1 \mathrm{~min}, 55^{\circ} \mathrm{C}$ for $1 \mathrm{~min}, 73^{\circ} \mathrm{C}$ for $1 \mathrm{~min}$ and a final extension step of $73^{\circ} \mathrm{C}$ for $10 \mathrm{~min}$. The Cys282Tyr mutation is due to a $G$ to $A$ transition in the exonic sequence (nucleotide 845 ) and this creates a second cleavage site for the restriction enzyme, Rsa1, in the amplified fragment. The PCR product from patient P1 was therefore digested with this restriction endonuclease, the products run on a polyacrylamide gel and the fragments visualised under UV light after staining with ethidium bromide (figure). Patient P1 was shown to be homozygous for the specific mutation. Four out of five other patients ( $\mathbf{P} 2$ to $\mathrm{P} 6$ ) with classical $\mathrm{HH}$ attending our Haematology clinic were also homozygous for the Cys282Tyr mutation. An analysis of 53 normal individuals gave an allele frequency of $4.7 \%(5 / 106)$ in the UK population (carrier frequency: $9.4 \%$ ).

\section{Discussion}

Asymptomatic close relatives of patients with $\mathrm{HH}$, particularly siblings, should be advised to 


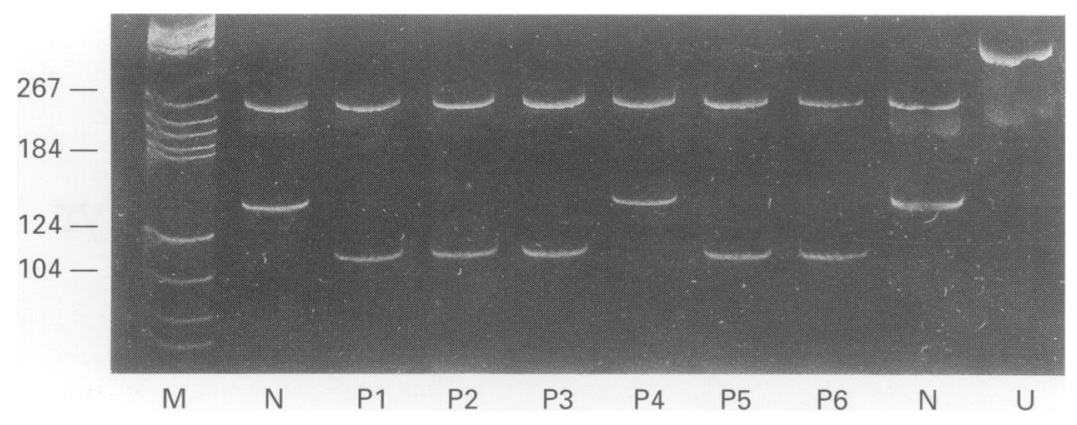

Figure Detection of Cys282Tyr mutation in the HLA-H gene by PCR and Rsal endonuclease digestion. The uncut fragment is approximately $400 \mathrm{bp}$ in size (U). An invariant Rsal site is present in the wild-type sequence giving fragments of about 260 and $140 \mathrm{bp}(\mathrm{N})$. Analysis of patient P1 and five other individuals (P2-P6) with haemochromatosis is shown. Patients homozygous for the Cys282Tyr mutation have an additional Rsal cleavage site in both alleles which produces fragments of 260,110 and $30 \mathrm{bp}$ (not shown). M: molecular size markers (pBR322 digested with HaeIII)

undergo screening for the disorder. ${ }^{1}$ Measurements of serum ferritin, serum iron and iron binding capacity have been used to detect iron overload in the first instance. Wide variation in these parameters may exist and additional tests are often required for example, linkage studies with HLA-A3 and other associated genetic markers. Occasional diagnostic difficulties may still arise, however, particularly in young women where there may be equivocal phenotypic parameters of iron overload. In such cases, liver biopsy for histochemical staining and chemical assay of liver iron may be required.

The identification of the $\mathrm{HH}$ gene, if confirmed, is a major advance in the accurate diagnosis of this common disorder. ${ }^{2} \mathrm{We}$ describe a modified technique for rapid PCRbased detection of the recurrent, candidate mutation (Cys282Tyr) found in $85 \%$ of $\mathrm{HH}$ patients thus far. In addition, the case presented demonstrates the usefulness of the test for diagnosis of patients in the early, asymptomatic stages of the disorder. A carrier frequency of $9.4 \%$ was demonstrated for the

\section{Summary/learning points}

- a candidate gene for hereditary haemochromatosis has been recently reported; $85 \%$ of patients are homozygous for a specific missense mutation which can be simply detected in the routine laboratory using the PCR

- the genetic test promises to be of value for rapid diagnosis of early asymptomatic cases and family screening

mutation in the UK population. This value is similar to previous estimates of the prevalence of the haemochromatosis gene in Caucasians based on phenotypic data. ${ }^{1}$

A number of uncertainties remain regarding the relationship between the HLA-H gene and $\mathrm{HH}$. Most fundamentally, proof is required that the gene is, in fact, identical to the putative $\mathrm{HH}$ gene rather than in tight linkage association with the disorder. If confirmed, it will be important to determine the nature and function of the HLA-H gene product in the context of iron metabolism and the mechanism by which alterations in the protein enhance iron accumulation. If not corroborated, however, the amino acid substitution may still prove to be useful for family studies as a closely linked genetic marker. Further information is also required in relation to the $15 \%$ of patients who do not carry the Cys282Tyr mutation. Preliminary evidence suggests that this subset of cases do not have alternative mutations in the HLA-H gene and lack genetic association with chromosome $6 \mathrm{p} .^{2}$

The detection of a frequent specific mutation in a candidate $\mathrm{HH}$ gene raises the possibility of population screening for this common disorder. The cost-benefit issues of such an undertaking require careful consideration but the significant morbidity encountered by patients in whom diagnosis is delayed and the simplicity of preventative therapy suggests that this topic should be a priority on the public health agenda.

1 Cox TM. Haemochromatosis. Blood Rev 1990; 4: 75-87.

2 Feder JN, Gnirke A, Thomas W, et al. A novel MHC class 1 -like gene is mutated in patients with hereditary haemochromatosis. Nat Genet 1996; 13: 399-408. 